Gynäkologische Endokrinologie 2013 .

11:161-161

DOI 10.1007/s10304-012-0529-z

Online publiziert: 15. August 2013

(c) Springer-Verlag Berlin Heidelberg 2013
T. Rabe ·T. Strowitzki

Universitäts-Frauenklinik Heidelberg

\section{Hormonelle Kontrazeption}

fragt hat. Es wurde klargestellt, dass sowohl das levonorgestrelhaltige als auch das ulipristalacetathaltige Notfallkontrazeptivum in der vorgegebenen Dosierung die Follikelreifung und den Eisprung unterdrückt bzw. verschiebt und keinen klinisch relevanten Einfluss auf die Implantation und die frühe Embryonalentwicklung hat. Das Kupfer-IUD, das man in Abhängigkeit vom Typ und der hierfür vorliegenden Zulassung ebenfalls bis zu 5 Tage postkoital einlegen kann, bietet dagegen zwar eine hohe kontrazeptive Sicherheit von $>99 \%$, wirkt aber implantationshemmend. Hierüber muss die Patientin im Einzelfall aufgeklärt werden.

Wir hoffen, Ihnen mit dieser aktuellen Übersicht über die derzeitigen Entwicklungen auf dem Gebiet der Kontrazeption eine kleine Hilfestellung für die tägliche Beratung an die Hand geben zu können.

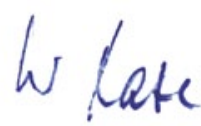

T. Rabe

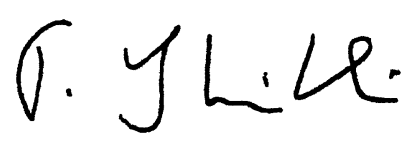

T. Strowitzki

\section{Korrespondenzadresse}

Prof. Dr. T. Rabe

Universitäts-Frauenklinik Heidelberg Im Neuenheimer Feld 440, 69120 Heidelberg thomas.rabe@med.uni-heidelberg.de 